Ibn Al-Haitham Jour. for Pure \& Appl. Sci. 33 (2) 2020

Ibn Al Haitham Journal for Pure and Applied Science

Journal homepage: http://jih.uobaghdad.edu.iq/index.php/j/index

\title{
Some Aspects of Weighted Rayleigh Distribution
}

Saad A. Zain

Department of mathematics, College of Education for Pure Science, Ibn Al

Haitham, University of Baghdad,

Baghdad, Iraq.

ssadadnan.2019@gmail.com
Iden Hassan Hussein

Department of Mathematics, College

of Science for Women, University of

Baghdad, Baghdad, Iraq

Iden_alkanani@yahoo.com

Article history: Received 3 June 2019, Accepted 15 July 2019, Published in April 2020.

Doi: $10.30526 / 33.2 .2431$

\section{Abstract}

In this paper, we proposed a new class of weighted Rayleigh distribution based on two parameters, scale and shape parameters which are introduced in Rayleigh distribution. The main properties of this class are investigated and derived.

Keywords: weighted Rayleigh distribution, quintile function skew-ness, and kurtosis.

\section{Introduction}

The Rayleigh distribution is an important distribution as a lifetime sample modeling. Many researchers developed various generalizations of Rayleigh probability density functions to increase the flexibility in life time sample modeling [1]. Introduced a new class of density functions depending on shape parameter in the normal distribution, and then called it weighted normal distribution or (skew-normal distribution). [2]. Used the idea of Azzalini to find the shape parameter to an exponential distribution and called it weighted exponential distribution, as well as put the general mathematical formula to treat the weighted statistical distributions which are as follow:

$$
f_{w}(X)=\frac{1}{\operatorname{Pr}\left[x_{2}<\theta x_{1}\right]} f\left(x_{1}\right) F\left(\theta x_{1}\right)
$$

Where $f_{w}(X)$ is weighted probability density function.

$f\left(x_{1}\right)$ isstandard probability density function for $r . v\left(x_{1}\right)$.

$f\left(\theta x_{1}\right)$ iscumulative distribution function with respect to weighted parameter $\theta$ for standard distribution.

$P_{r}\left(x_{2}<\theta x_{1}\right)$ probability for $r . v\left(x_{2}\right)$ with respect to the $r . v\left(x_{1}\right)$ and weighted function $(\theta)$. [3]. Study weighed Weibull distribution by using the idea of Azzalini, and introduce the basic properties for this model. [4]. Study the skew-ness parameter of a gamma distribution by employing the idea of Azzalini, which creates a new class of weighed gamma distribution. [5]. Propose the extension of Weibull distribution weighted and (the main derived) main 
properties of this class are derived [6]. Estimate Linley's approximation method for weighted exponential distribution by using Monte Carlo simulation study. [7]. Introduce two shape parameters to the existing weighted exponential distribution to develop the Beta weighted exponential distribution using the legit of beta function.[8]. propose model named exponentiated weighted exponential distribution and some of the basic statistical properties of the proposed model are studied and provided. [9]. Focus on Bayes estimation of weighed exponential distribution with fuzzy data. [10]. Derive two parameters inverted weighted exponential distribution and its various statistical properties are established. [11]. Present a new generalization weighted Weibull distribution. The aim of this paper is to propose a new weighted Rayleigh distribution depending on the idea of Azzalini and apply the general formula of Gupta and Kundu in equation (1) to find the skew ness parameter in Rayleigh density function, then study some basic statistical properties of this distribution. The rest of this article is organized as follows: we include in section two the weighted Rayleigh distribution with application, Cumulative distribution function, reliability function and hazard function. In section three, the moment, mean, and variance. In section four, the moment generating functions. In section five, the factorial moment generating function, skew ness kurtosis, and characteristic-function and in section six, the quintile function, mode and median.

\section{Weighted Rayleigh Distribution}

In this section, we introduce of the probability density function and the cumulative distribution function of weighted Rayleigh distribution (WRD).

\section{Lemma (1)}

Suppose that $x_{1}$ and $x_{2}$ are random sample of size two of Rayleigh distribution with parameter $\theta$, then the $\operatorname{pr}\left[\alpha x_{1}>x_{2}\right]=\frac{\alpha^{2}}{\alpha^{2}+1}$ where $\frac{\alpha^{2}+1}{\alpha^{2}}$ is weighted Rayleigh distribution.

Proof:

$$
\begin{aligned}
& \operatorname{pr}\left[\alpha x_{1}>x_{2}\right]=\operatorname{pr}\left[x_{2}<\alpha x_{1}\right] \\
& \operatorname{pr}\left[x_{2}<\alpha x_{1}\right]=\int_{\text {all } x_{1}} \int_{\text {all } x_{2}} f\left(x_{1}, x_{2}\right) d x_{2} d x_{1} \\
& \operatorname{pr}\left[x_{2}<\alpha x_{1}\right]=\int_{0}^{\alpha x_{1}} \int_{0}^{\infty} \theta x_{1} e^{-\frac{\theta}{2} x_{1}^{2}} \theta x_{2} e^{-\frac{\theta}{2} x_{2}^{2}} d x_{2} d x_{1} \\
& \operatorname{pr}\left[x_{2}<\alpha x_{1}\right]=\int_{0}^{\infty} \theta x_{1} e^{-\frac{\theta}{2} x_{1}^{2}}\left[\int_{0}^{\alpha x_{1}} \theta x_{2} e^{-\frac{\theta}{2} x_{2}^{2}} d x_{2}\right] d x_{1} \\
& \operatorname{pr}\left[x_{2}<\alpha x_{1}\right]=\int_{0}^{\infty} \theta x_{1} e^{-\frac{\theta}{2} x_{1}^{2}}\left[-\left.e^{-\frac{\theta}{2} x_{2}^{2}}\right|_{0} ^{\alpha x_{1}}\right] d x_{1} \\
& \operatorname{pr}\left[x_{2}<\alpha x_{1}\right]=\int_{0}^{\infty} \theta x_{1} e^{-\frac{\theta}{2} x_{1}^{2}}\left[1-e^{--\frac{\theta}{2} \alpha^{2} x_{1}^{2}}\right] d x_{1} \\
& \operatorname{pr}\left[x_{2}<\alpha x_{1}\right]=\int_{0}^{\infty} \theta x_{1} e^{-\frac{\theta}{2} x_{1}^{2}} d x_{1}-\int_{0}^{\theta} \theta x_{1} e^{-\frac{\theta}{2} x_{1}^{2}\left(\alpha^{2}+1\right)} d x_{1}
\end{aligned}
$$




$$
\begin{aligned}
& \operatorname{pr}\left[x_{2}<\alpha x_{1}\right]=1-\left[\frac{-1}{\alpha^{2}+1} \int_{0}^{\infty} \theta x_{1}\left(\alpha^{2}+1\right) e^{-\frac{\theta}{2} x_{1}^{2}\left(\alpha^{2}+1\right)} d x_{1}\right] \\
& \operatorname{pr}\left[x_{2}<\alpha x_{1}\right]=1-\left[\frac{1}{\alpha^{2}+1}\right]=\frac{\alpha^{2}}{\alpha^{2}+1} \\
& \frac{1}{\operatorname{pr}\left[x_{2}<\alpha x_{1}\right]}=\frac{1}{\frac{\alpha^{2}}{\alpha^{2}+1}}=\frac{\alpha^{2}+1}{\alpha^{2}}
\end{aligned}
$$

Then the proposed new weighted Rayleigh distribution is defined as follows:

$f(x ; \alpha, \theta)=\frac{\alpha^{2}+1}{\alpha^{2}} \theta x e^{-\frac{\theta}{2} x^{2}}\left[1-e^{-\frac{\theta}{2} x^{2} \alpha^{2}}\right]$

Now, proving that the weighted Rayleigh distribution is probability density function as follows:

$$
\begin{aligned}
& \int_{0}^{\infty} \frac{\alpha^{2}+1}{\alpha^{2}} \theta x e^{-\frac{\theta}{2} x^{2}}\left[1-e^{-\frac{\theta}{2} x^{2} \alpha^{2}}\right] d x=1 \\
& \frac{\alpha^{2}+1}{\alpha^{2}}\left\{\int_{0}^{\infty} \theta x e^{-\frac{\theta}{2} x^{2}} d x-\int_{0}^{\infty} \theta x e^{\frac{\theta}{2} x^{2}\left(\alpha^{2}+1\right)} d x\right\} \\
& =\frac{\alpha^{2}+1}{\alpha^{2}}\left\{1-\frac{1}{\alpha^{2}+1}\right\}=\frac{\alpha^{2}+1}{\alpha^{2}} * \frac{\alpha^{2}}{\alpha^{2}+1}=1
\end{aligned}
$$

\section{Lemma (2)}

The cumulative distribution function of weighted Rayleigh distribution is as follows:

Proof:

$F(x ; \alpha, \theta)=\operatorname{Pr}[X \leq x]=\int_{0}^{x} f(u) d u$

$F(x ; \alpha, \theta)=\int_{o}^{x} \frac{\left(\alpha^{2}+1\right)}{\alpha^{2}} \theta u e^{-\frac{\theta}{2} u^{2}}\left(1-e^{-\frac{\theta}{2} u^{2} \alpha^{2}}\right) d u$

$F(x ; \alpha, \theta)=\frac{\alpha^{2}+1}{\alpha^{2}}\left\{\frac{\left(\alpha^{2}+1\right)-\left(\alpha^{2}+1\right) e^{-\frac{\theta}{2} x^{2}}+e^{-\frac{\theta}{2} x^{2}\left(\alpha^{2}+1\right)}-1}{\alpha^{2}+1}\right\}$

$F(x ; \alpha, \theta)=\frac{\alpha^{2}+1}{\alpha^{2}}-\frac{\left(\alpha^{2}+1\right) e^{-\frac{\theta}{2} x^{2}}}{\alpha^{2}}+\frac{e^{-\frac{\theta}{2} x^{2}\left(\alpha^{2}+1\right)}}{\alpha^{2}}-\frac{1}{\alpha^{2}}$

$F(x ; \alpha, \theta)=1-\left[\frac{\left(\alpha^{2}+1\right) e^{-\frac{\theta}{2} x^{2}}-e^{-\frac{\theta}{2} x^{2}\left(\alpha^{2}+1\right)}}{\alpha^{2}}\right]$

The reliability and hazard functions are as follows:

$$
\begin{aligned}
& R(x)=1-F(x) \\
& R(x)=\frac{\left(\alpha^{2}+1\right) e^{-\frac{\theta}{2} x^{2}}-e^{-\frac{\theta}{2} x^{2}\left(\alpha^{2}+1\right)}}{\alpha^{2}} \\
& h(x)=\frac{f(x)}{R(x)}
\end{aligned}
$$


$h(x)=\frac{\theta x\left(\alpha^{2}+1\right)\left[1-e^{-\frac{\theta}{2} x^{2} d^{2}}\right]}{\left[\left(\alpha^{2}+1\right)-e^{-\frac{\theta}{2} x^{2} \alpha^{2}}\right]}$

\section{Moment Method}

Now, we derived the moment a bout zero, then we find the mean and the variance of the Weighted Rayleigh distribution which is as follows:

$$
\begin{aligned}
& E\left(X^{r}\right)=\int_{o}^{\infty} x^{r} \frac{\alpha^{2}+1}{\alpha^{2}} \theta x e^{-\frac{\theta}{2} x^{2}}\left[1-e^{-\frac{\theta}{2} x^{2 \alpha^{2}}}\right] d x \\
& E\left(X^{r}\right)=\frac{\alpha^{2}+1}{\alpha^{2}}\left\{\int_{0}^{\infty} x^{r+1} \theta e^{-\frac{\theta}{2} x^{2}} d x-\int_{0}^{\infty} x^{r+1} \theta e^{-\frac{\theta}{2} x^{2\left(\alpha^{2}+1\right)}} d x\right\} \\
& \text { let } y=\frac{\theta}{2} x^{2}, x=\left(\frac{2}{\theta}\right)^{\frac{1}{2}} y^{\frac{1}{2}}, d x=\left(\frac{2}{\theta}\right)^{\frac{1}{2}} \frac{1}{2 \sqrt{y}} d y \\
& \text { lt } u=\frac{\theta}{2} x^{2}\left(\alpha^{2}+1\right), x=\left(\frac{2}{\theta\left(\alpha^{2}+1\right)}\right)^{\frac{1}{2}} u^{\frac{1}{2}}, d x=\left(\frac{2}{\theta\left(\alpha^{2}+1\right)}\right)^{\frac{1}{2}} \frac{1}{2 \sqrt{u}} d u \\
& E\left(x^{r}\right)=\frac{\alpha^{2}+1}{\alpha^{2}}\left\{\int_{o}^{\infty}\left[\left(\frac{2}{\theta}\right)^{\frac{1}{2}} y^{\frac{1}{2}}\right]^{r+1} \theta e^{-y}\left(\frac{2}{\theta}\right)^{\frac{1}{2}} \frac{1}{2 \sqrt{y}} d y\right. \\
& \left.-\int_{o}^{\infty}\left[\left(\frac{2}{\theta\left(\alpha^{2}+1\right)}\right)^{\frac{1}{2}} u^{\frac{1}{2}}\right]^{r+1} \theta e^{-u}\left(\frac{2}{\theta\left(\alpha^{2}+1\right)}\right)^{\frac{1}{2}} \frac{1}{2 \sqrt{u}} d u\right\} \\
& E\left(X^{r}\right)=\frac{\alpha^{2}+1}{\alpha^{2}}\left\{\int_{o}^{\infty}\left(\frac{2}{\theta}\right)^{\frac{r}{2}} y^{\frac{r}{2}}\left(\frac{2}{\theta}\right)^{\frac{1}{2}} y^{\frac{1}{2}} \theta e^{-y}\left(\frac{2}{\theta}\right)^{\frac{1}{2}} \frac{1}{2} y^{-\frac{1}{2}} d y\right. \\
& \left.-\int_{0}^{\infty}\left(\frac{2}{\theta\left(\alpha^{2}+1\right)}\right)^{\frac{r}{2}}\left(\frac{2}{\theta\left(\alpha^{2}+1\right)}\right)^{\frac{1}{2}} u^{\frac{r}{2}} u^{\frac{1}{2}} \theta e^{-u}\left(\frac{2}{\theta\left(\alpha^{2}+1\right)}\right)^{\frac{1}{2}} \frac{1}{2} u^{-\frac{1}{2}} d u\right\} \\
& E\left(X^{r}\right)=\frac{\alpha^{2}+1}{\alpha^{2}}\left\{\left(\frac{2}{\theta}\right)^{\frac{r}{2}} \int_{o}^{\infty} \frac{2}{\theta} y^{\frac{r}{2}} \theta e^{-y} \frac{1}{2} d y-\left(\frac{2}{\theta\left(\alpha^{2}+1\right)}\right)^{\frac{r}{2}} \int_{o}^{\infty} \frac{2}{\theta\left(\alpha^{2}+1\right)} u^{\frac{r}{2}} \theta e^{-u} \frac{1}{2} d u\right. \\
& E\left(X^{r}\right)=\frac{\alpha^{2}+1}{\alpha^{2}}\left\{\left(\frac{2}{\theta}\right)^{\frac{r}{2}} \Gamma\left(\frac{r}{2}+1\right)-\left(\frac{2}{\theta}\right)^{\frac{r}{2}} \frac{1}{\left(\alpha^{2}+1\right)^{\frac{r}{2}+1}} \Gamma\left(\frac{r}{2}+1\right)\right\} \\
& E\left(X^{r}\right)=\frac{\alpha^{2}+1}{\alpha^{2}}\left(\frac{2}{\theta}\right)^{\frac{r}{2}} \Gamma\left(\frac{r}{2}+1\right)\left[1-\frac{1}{\left(\alpha^{2}+1\right)^{\frac{r}{2}+1}}\right]
\end{aligned}
$$

When $r=1$, then

$$
E(X)=\frac{\alpha^{2}+1}{\alpha^{2}}\left(\frac{2}{\theta}\right)^{\frac{1}{2}} \Gamma\left(\frac{3}{2}\right)\left[1-\frac{1}{\left(\alpha^{2}+1\right)^{3 / 2}}\right]
$$




$$
\begin{aligned}
& \mathrm{E}(\mathrm{X})=\frac{\alpha^{2}+1}{\alpha^{2}}\left(\frac{2}{\theta}\right)^{\frac{1}{2}} \frac{1}{2} \sqrt{\pi}\left[1-\frac{1}{\left(\alpha^{2}+1\right)^{\frac{3}{2}}}\right] \\
& \mathrm{E}(\mathrm{X})=\frac{\alpha^{2}+1}{\alpha^{2}}\left[\frac{\pi}{2 \theta}\right]^{\frac{1}{2}}\left[1-\frac{1}{\left(\alpha^{2}+1\right)^{3 / 2}}\right]
\end{aligned}
$$

when $r=2$, then

$$
\begin{aligned}
& E\left(X^{2}\right)=\frac{\alpha^{2}+1}{\alpha^{2}}\left(\frac{2}{\theta}\right) \Gamma(2)\left[1-\frac{1}{\left(\alpha^{2}+1\right)^{2}}\right] \\
& E\left(X^{2}\right)=\frac{2\left(\alpha^{2}+2\right)}{\theta\left(\alpha^{2}+1\right)}
\end{aligned}
$$

The variance:

$$
\begin{aligned}
& \operatorname{var}(X)=E\left(X^{2}\right)-[E(X)]^{2} \\
& \operatorname{var}(X)=\frac{2\left(\alpha^{2}+2\right)}{\theta\left(\alpha^{2}+1\right)}-\frac{\left(\alpha^{2}+1\right)^{2}}{\alpha^{4}} \frac{\pi}{2 \theta}\left[1-\frac{1}{\left(\alpha^{2}+1\right)^{\frac{3}{2}}}\right]^{2} \\
& \operatorname{var}(X)=\frac{4 \alpha^{4}\left(\alpha^{2}+2\right)-\pi\left[\left(\alpha^{2}+1\right)^{\frac{3}{2}}-1\right]^{2}}{2 \theta \alpha^{4}\left(\alpha^{2}+1\right)}
\end{aligned}
$$

\section{4-The Moment Generated Function}

We derived the moment generating function for weighted Rayleigh distribution which is as follows:

m.g.f. $=E\left[e^{t X}\right]=\int_{0}^{\infty} e^{t x} f(x) d x$

By using Taylor series:

$E\left(e^{t X}\right)=\int_{0}^{\infty}\left(1+\frac{t x}{1 !}+\frac{t x^{2}}{2 !}+\frac{t x^{3}}{3 !}+\cdots+\frac{t x^{r}}{r !}\right) f(x) d x$

$m . g . f=\sum_{r=0}^{\infty} \frac{t^{r}}{r !} E\left(x^{r}\right)$

m.g.f $=\sum_{r=0}^{\infty} \frac{t^{r}}{r !} \frac{\alpha^{2}+1}{\alpha^{2}}\left(\frac{2}{\theta}\right)^{\frac{r}{2}} \Gamma\left(\frac{r}{2}+1\right)\left[1-\frac{1}{\left(\alpha^{2}+1\right)^{r / 2}+1}\right]$

m.g.f $=\frac{\alpha^{2}+1}{\alpha^{2}} \sum_{r=0}^{\infty} \frac{t^{r}}{r !}\left(\frac{2}{\theta}\right)^{\frac{r}{2}} \Gamma\left(\frac{r}{2}+1\right)\left[1-\frac{1}{\left(\alpha^{2}+1\right)^{r} / 2+1}\right]$

\section{5- Factorial Moment Generating Function:}

$$
\begin{aligned}
& M_{x}(t)=E\left(t^{x}\right)=\int_{0}^{\infty} t^{x} f(x) d x \\
& M_{x}(t)=\int_{0}^{\infty} e^{\ell n t^{x}} f(x) d x
\end{aligned}
$$




$$
\begin{aligned}
& M_{x}(t)=\int_{0}^{\infty}\left(1+x \ell n t+\frac{x^{2}(\ell n t)^{2}}{2 !}+\cdots+\frac{x^{r}(\ell n t)^{r}}{r !}+\cdots \ldots . .\right) f(x) d x \\
& M_{x}(t)=\sum_{r=0}^{\infty} \frac{(\ell n t)^{r}}{r !} E\left(x^{r}\right) \\
& M_{x}(t)=\frac{\alpha^{2}+1}{\alpha^{2}} \sum_{r=0}^{\infty} \frac{(\ell n t)^{r}}{r !}\left(\frac{2}{\theta}\right)^{\frac{r}{2}} \Gamma\left(\frac{r}{2}+1\right)\left[1-\frac{1}{\left(\alpha^{2}+1\right)^{\frac{r}{2}+1}}\right]
\end{aligned}
$$

Then the Coefficient of skew ness of this distribution is as follows:

$$
\begin{aligned}
& \left.\left.C . S=\frac{M_{3}}{\left(M_{2}\right)^{\frac{3}{2}}}, M_{3}=E(X-E(X))\right)^{3}, M_{2}=E(X-E(X))\right)^{2} \\
& \text { C. } S=\frac{E(X-E(X))) 3,}{E(X-E(X)))_{3}^{3}}=1 \\
& \text { C. } K=\frac{M_{4}}{\left(M_{2}\right)^{2}}=\frac{E(X-E(X)))^{4}}{(E(X-E(X)))^{2}}
\end{aligned}
$$

\section{The characteristic function of this distribution is as follow:}

$$
\begin{aligned}
& Q_{X}(t)=E\left(e^{i t x}\right)=\int_{0}^{\infty} e^{i t x} f(x) d x \\
& Q_{X}(t)=\int_{0}^{\infty}\left(1+i t x+\frac{(i t)^{2} x^{2}}{2 !}+\cdots \frac{(i t)^{r} x^{r}}{r !}\right) f(x) d x \\
& Q_{X}(t)=\sum_{r=0}^{\infty} \frac{(i t)^{r}}{r !} E\left(X^{r}\right) \\
& Q_{X}(t)=\sum_{r=0}^{\infty} \frac{(i t)^{r}}{r !} \frac{\alpha^{2}+1}{\alpha^{2}}\left(\frac{2}{\theta}\right)^{r} \Gamma\left(\frac{r}{2}+1\right)\left[1-\frac{1}{\left(\alpha^{2}+1\right)^{\frac{r}{2}+1}}\right] \\
& Q_{X}(t)=\frac{\alpha^{2}+1}{\alpha^{2}} \sum_{r=0}^{\infty} \frac{(i t)^{r}}{r !}\left(\frac{2}{\theta}\right)^{r} \Gamma\left(\frac{r}{2}+1\right)\left[1-\frac{1}{\left(\alpha^{2}+1\right)^{\frac{r}{2}+1}}\right]
\end{aligned}
$$

\section{6-Quintile Function}

The quintile of this distribution is denoted by $x=F^{-1}(v), \mathrm{v}=F(x)$ :

$$
\begin{aligned}
& F(x)=1-\left[\frac{(\alpha+1) e^{-\frac{\theta}{2} x^{2}}-e^{-\frac{\theta}{2} x^{2}\left(\alpha^{2}+1\right)}}{\alpha^{2}}\right] \\
& V=1-\left[\frac{\left(\alpha^{2}+1\right) e^{-\frac{\theta}{2} x^{2}}-e^{-\frac{\theta}{2} x^{2}\left(\alpha^{2}+1\right)}}{\alpha^{2}}\right]
\end{aligned}
$$


$e^{-\frac{\theta}{2} x^{2}}\left[\left(\alpha^{2}+1\right)-e^{-\frac{\theta}{2} x^{2} \alpha^{2}}\right]-\alpha^{2}(1-v)=0$

The equation (15) is difficult to solve, and then, to solve it is by using the numerical methods after compensation the estimate of $\theta, \alpha$ in it. To find the mode of this distribution we apply $f^{\prime}(x)=0$ which is as follows:

$$
\begin{aligned}
& \text { Let } K=\frac{\alpha^{2}+1}{\alpha^{2}} \\
& \ln f(x)=\ln (K)+\ln \theta+\ln x-\frac{\theta}{2} x^{2}+\ln \left[1-e^{-\frac{\theta}{2} x^{2} \alpha^{2}}\right] \\
& \frac{d \ell n f(x)}{d x}=\frac{1}{x}-\theta x+\frac{\theta x \alpha^{2} e^{-\frac{\theta}{2} x^{2} \alpha^{2}}}{1-e^{-\frac{\theta}{2} x^{2} \alpha^{2}}} \\
& \frac{d \ln f(x)}{d x}=0, \quad \frac{1}{x}-\theta x+\frac{\theta x \alpha^{2} e^{-\frac{\theta}{2} x^{2} \alpha^{2}}}{1-e^{-\frac{\theta}{2} x^{2} \alpha^{2}}}=0 \\
& e^{-\frac{\theta}{2} x^{2} \alpha^{2}}\left[1-\theta x^{2}-\theta x^{2} \alpha^{2}\right]-\left(1-\theta x^{2}\right)=0
\end{aligned}
$$

The equation (16) can be solved using numerical methods because it very difficult.

To find the median of this distribution, we apply $F(x)=\frac{1}{2}$ which is as follows:

$$
\begin{aligned}
& F(x)=\frac{1}{2} \\
& 1-\left[\frac{\left(\alpha^{2}+1\right) e^{-\frac{\theta}{2} x^{2}}-e^{-\frac{\theta}{2} x^{2}\left(\alpha^{2}+1\right)}}{\alpha^{2}}\right]=\frac{1}{2} \\
& e^{-\frac{\theta}{2} x^{2}}\left[\left(\alpha^{2}+1\right)-e^{-\frac{\theta}{2} x^{2}\left(\alpha^{2}+1\right)}\right]-0.5 \alpha^{2}=0
\end{aligned}
$$

The equation (17) solves it by using numerical methods.

\section{Conclusion}

Weighted distributions are employed mostly in study of reliability, bio-medicine, metaanalysis, econometrics, survival analysis, renewal processes, physics, ecology and branching processes. A weighted model based on the Rayleigh distribution is proposed in this work and the statistical properties of this model are presented. Some non-Bayesian and Bayesian methods may be used to estimate the parameter of proposed model in the future work. Furthermore, the proposed model may be employed for a real data depending on accurate goodness-of-fit test

\section{Acknowledgments}

We would like to thank the referee for valuable and constructive comments which significantly improved the paper.

\section{References}

1. Azzalini, A. A Class of Distributions, which includes. The normal ones, Board of the foundation of the Scandinavian journal of statistics.1985, 12, 2,171-178.

2. Gupta, R.D.; Kundu, D. A new class of weighted exponential distributions. Statistics.2009, 43, 6, pp.621-634.

3. Shahbaz, S.; Shahbaz, M.Q.; Butt, N.S. A class of weighted Weibull distribution. Pak. j. stat. oper. Res.2010, 6, 1, 53-59. 
4. Ramadan, Mervat, Mahdy. A class of weighed Gamma Distributions its properties, Economic Quality Control.2010, 26 , 2, 133-144.

5. Essam, A.A.; Mohamed, A.H. A weighted three-parameter Weibull distribution. J. Applied Sci. Res.2013, 9, 6627-6635.

6. Farahani, Z.S.M.; Khorram, E. Bayesian statistical inference for weighted exponential distribution. Communications in Statistics-Simulation and Computation.2014, 43, 6, 1362-1384.

7. Badmus, N.I.; Bamiduro. T. Adebayo, Animasaun.A Monsuru,and Akingbade.A Akkeem, The Beta weighted exponential distribution. Theory and Application, International journal of mathematical Analysis and optimization. 55-66.

8. Oguntunde, p.E. On the Exponentiated Weighted Exponential Distribution and its Basic statistical properties. PSCI Publication.2015, 10, 3, 160-167.

9. Al-Noor, N.H.; Hussein, L.K. Weighted Exponential Distribution: Approximate Bayes Estimations with Fuzzy Data. Al-Nahrain Journal of Science.2018, 1, 174-185.

10. Jabber, A.K.; Tawfiq, L.N.M. New Transform Fundamental Properties and its Applications. Ibn Alhaitham Journal for Pure and Applied Science.2018, 31, 1, 151163.

11. Salman, Abbes.; et.al A new generalized Weightd Weibull Distribution, Pakistan journal of statistics and operation Research.2019, 15, 1,161- 178. 